\title{
Recognition of complex object-centred spatial configurations in early infancy
}

\author{
Andrew Bremner ${ }^{1,2}$, Peter Bryant ${ }^{3}$, Denis Mareschal ${ }^{2} \&$ Ágnes Volein ${ }^{2}$ \\ ${ }^{1}$ Department of Psychology, Goldsmiths, University of London \\ ${ }^{2}$ Centre for Brain and Cognitive Development, Birkbeck, University of London \\ ${ }^{3}$ Department of Educational Studies, University of Oxford
}

Running Head: Object recognition in infants

Keywords: object recognition, spatial representation, infancy, cognitive development

Address for correspondence: Department of Psychology, Whitehead Building,

Goldsmiths, University of London, New Cross, London, SE14 6NW. United Kingdom.

Email: a.bremner@gold.ac.uk

Telephone: +44 (0) 2070785074

Fax: +44 (0) 2079197873 
Acknowledgements

This research was supported by a University Scholarship from the University of Oxford, and a postdoctoral research fellowship from the ESRC (T026271357), both awarded to AB. Additional support was provided by European Commission grants HPRN-CT-2000-00065 and NEST (516542). The authors would like to thank Jordy Kaufman for assistance in programming, Leslie Tucker for assistance in data collection, and also Oliver Braddick, Brian Rogers, Alan Slater \& an anonymous reviewer for helpful comments on earlier drafts of this manuscript. 


\begin{abstract}
In a series of experiments we tested 4- and 8-month-olds' ability to represent the spatial layout of an object across changes in its orientation with respect to egocentric spatial coordinates. A fixed-trial familiarisation procedure based on visual habituation behaviour shows that both age-groups are able to discriminate between different object-centred spatial configurations. Furthermore, both age-groups demonstrate the ability to make discriminations of object-centred spatial coordinates that require simultaneous reference to at least two spatial axes of the object. We discuss these findings in relation to theories of the early development of object recognition and spatial reference skills.
\end{abstract}


Recognition of complex object-centred spatial configurations in early infancy

The origin of the human object concept continues to be a key topic for debate in developmental psychology. An important component of the object concept, and one that was emphasised in Piaget's (1937/1954) writings, is an appreciation of object constancy; that is, the understanding that some properties of objects remain invariant despite the various changes in proximal (retinal) stimulation that are caused by movement of both objects and observers. Appreciation of an object's constancy involves an understanding that it has a constant shape despite changes in orientation, a constant size despite changes in its distance, and continued existence (or permanence) despite its occlusion.

One particularly important aspect of infants' object concept development that has received relatively little attention so far is the ability to represent and recognise an object's constant spatial layout across changes in its orientation. Objects (especially manipulable objects), as well as moving in depth, frequently change in their orientation relative to the observer and environment. As such, they do not retain a fixed spatial relation to either egocentric or environmental frames of reference. Thus, in order to represent the spatial relations of features within objects, infants need to utilise a spatial frame of reference that is independent of retinocentric, egocentric, and allocentric coordinates ${ }^{1}$. Marr (1980) termed such spatial representations in adults 'object-centred', and claimed that these mental structures formed the basis of our object recognition abilities.

Our current understanding of young infants' competence at recognising objects across changes in orientation and distance has been gleaned indirectly from research into size and shape constancy. Slater and colleagues have shown that newborns can discriminate between objects on the basis of their real shape despite 
changes in slant (in depth) relative to the observer's retina (Slater \& Morison; 1985), and also their real size despite changes in their distance from the retina (Slater, Mattock \& Brown; 1990).

Recently however, Bremner, Bryant \& Mareschal (2006) have tackled the problem of object-centred spatial representation more directly. Using a fixed-trial familiarisation procedure similar to that employed by Slater et al $(1983,1990)$ they familiarised eighteen 4-month-old infants to a specific spatial configuration within an object across six different orientations within the frontal plane. On subsequent test trials the object was presented to the infants in an entirely novel orientation. Between successive test trials the within-object spatial configuration was alternated between novel and familiar. The infants demonstrated a significant visual preference for the novel object-centred spatial configuration, indicating that by 4 months of age, infants can represent the spatial relation of a feature to an object-centred frame of reference.

In order to understand the basis of this competence, it is important to determine what representations underlie the infants' success at this task. The representations underlying adults' recognition of object-centred spatial configurations is a question of continued controversy (Biederman, 1987; Hummel, 2000; Mozer, 2001; Tarr, Williams, Hayward \& Gautier, 1999; Tipper and Behrmann, 1996; Vecera, Behrmann \& Filapek, 2001). One view is that objects are encoded and stored relative to egocentric spatial coordinates (Tarr, 1999), and that the information in such egocentric representations is rich enough to provide reliable object recognition across a variety of changes in orientation relative to the observer (Mozer, 2001). In contrast, following Marr and Nishihara (1978), Biederman (1987) has argued that certain '3D volumes' (or parts of objects) can be described by the visual system in a view invariant code. 
Support for these 'structural description' theories has come from neuropsychological evidence of specific impairments in object-centred spatial representation (Tipper \& Behrmann, 1996), and evidence for object- and part-guided attention in adults (Tipper, Driver \& Weaver, 1991; Hummel, 2001; Vecera, Behrmann \& Filapek, 2001), and object-guided attention in 8-month-old infants (Johnson \& Gilmore, 1999). Despite strong objections to a pure view-invariant code for object recognition (Tarr, 1999; Tarr and Bulthoff, 2001) there is still clear agreement that at least some degree of object-centred spatial representation exists (Mozer, 2001; Tarr \& Pinker, 1990). Indeed, the mature visual system may use both view-specific and view-invariant representations of objects (Hummel, 2001).

One particularly important approach to characterising adult object recognition is to identify when viewer- or object-centred representations are employed. Since Shepard \& Metzler (1971), the use of view-centred codes in object recognition has been identified by measuring the speed of adult participants' recognition of objects across changes in orientation. If the speed of recognition is affected by difference in orientation, this is taken to imply that mental rotation is used to match objects against egocentrically/environmentally defined spatial maps (Shepherd \& Metzler, 1971; Tarr, Williams, Hayward, \& Gautier, 1998).

Using this paradigm, Tarr \& Pinker (1990) asked whether the complexity of the object-centred spatial relations required for distinguishing between objects has an affect on the choice of spatial code employed in recognition. They gave adults the task of learning names for a set of three novel abstract shapes, and then timed the participants at naming reoriented versions of the same set of shapes. All of the shapes were composed of the same local features so that recognition required the processing of a global configuration. Four groups of participants were given different kinds of 
shapes to recognise. In three of these groups discrimination of the shapes across changes in orientation required spatial reference to only one axis (or dimension) of the object-centred framework. However, the fourth group required coordinated reference to two axes of the object-centred framework. Tarr and Pinker (1990) found that the time that the subjects took to recognise these shapes was constant across the degree of reorientation of the shapes in all conditions bar the two axis condition indicating that mental rotation was used in this condition only.

Tarr \& Pinker (1990) concluded that object recognition tasks requiring a representation of features relative to more than one axis of the object-centred framework are solved by mentally rotating an image of the object to match against a learned viewer-centred/egocentric representation. Thus, it seems that a mature object concept is characterised by, on the one hand, at least some ability to represent objectcentred spatial relations independently of the egocentric and environmental frameworks, but also by an ability to represent changes in the orientation of the object with respect to the egocentric/environmental spatial array.

The object-centred spatial discriminations presented to 4-month-old infants by Bremner et al (2006) only required 1-dimensional spatial reference for recognition. As detailed above, Tarr and Pinker (1990) have shown that adults do not usually form object-centred representations that coordinate features relative to two axes of the object-centred spatial framework - and in these situations resort to viewer-centred recognition strategies. In this article we examine infants' ability to make discriminations between objects that are differentiated at more than one level of object-centred spatial complexity. We achieve this by manipulating the number of object-centred axes that must be coordinated in representation in order to discriminate these configurations across a change in orientation (as in Tarr and Pinker, 1990). 
--Figure 1 about here--

The T-shape (see Figure 1) provides an easy way of contrasting discriminations of object-centred configurations that require one- or two-dimensional spatial reference. In order to distinguish between 'object-centred locations' (OCLs) 1 and 2, or between OCL 1 and 3, by the use of object-centred coordinates alone, representations of only one spatial axis is required, as the configurations of light to object differ with respect to both of the object's axes (within the picture plane). We will call this a '1D discrimination'. However, in order to distinguish between OCLs 2 and 3 , in reference to the object-centred framework alone, an observer is required to coordinate representations of the light's spatial relation to two axes of the object. We will call this a '2D discrimination'.

Figure 2 illustrates the distinction between 1D and 2D discriminations. The two objects in each figure are disoriented from each other with respect to the egocentric spatial array. The $1 \mathrm{D}$ discrimination only requires the representation of OCL within single axes of the object-centred frame of reference. However, in the 2D OCL discrimination (see Figure 2b) OCLs 2 and 3 are distinguished by coordinating spatial information from two (picture plane) axes of the object (the $\mathrm{x}$ and $\mathrm{y}$ axes in the figure). The spatial predicates required for representing the two-dimensional spatial structure of the object are necessarily more complicated than those required for representing a single spatial axis of the object.

--Figure 2 about here--

The current experiments examine object-centred spatial representation competence across two age groups of infants. In order to investigate the complexity of object-centred spatial representation available to these age-groups we presented both groups with 1D and 2D discriminations. Experiment 1 compares 4-month-olds' 
and 8-month-olds' ability to represent in memory and discriminate object-centred spatial relations at two degrees of complexity (1D and 2D). Experiments $2 a$ and $2 b$ make a minor procedural modification to the familiarisation procedure employed in Experiment 1 in order to test 4-month-olds' ability represent and discriminate 2D object-centred spatial configurations more fairly.

\section{Experiment 1}

Experiment 1 examines 4- and 8-month-olds' ability to make 1D and 2D OCL discriminations. Infants' are first familiarised to one object-centred spatial configuration across six different object orientations. Each of the six familiarisation trials last until the infant being tested has accumulated 15 seconds (Quinn, Slater, Brown \& Hayes, 2001) of looking at the object. After familiarisation they are then presented with two test trials in which novel and familiar object-centred configurations are presented side by side in novel orientations.

\section{Method}

\section{$\underline{\text { Stimuli }}$}

The target object was shaped in the form of a capital ' $\mathrm{T}$ ', and thus comprised three limbs; one perpendicularly oriented in relation to the other two. All three limbs of the object were identical apart from their spatial relation to the other limbs. Each limb also contained a marked location that was occupied by a light that could be switched on or off (labelled as OCLs 1 to 3 in Figure 1). When illuminated these object-centred locations were identical in appearance, making it possible for us to manipulate the spatial relation of a feature (light) to the object framework, simply by changing the OCL that was illuminated.

The object's orientation could be changed by rotating it within the frontal plane around the central 'point of rotation' labelled in Figure 1. By rotating the object 
we were able to present any one object-centred location in many different egocentric/allocentric locations. As each of the OCLs was equidistant from the point of rotation, each also had the capacity to occupy the same distribution of locations in egocentric/allocentric space.

\section{$\underline{\text { Design }}$}

In order to avoid confounding object-centred with egocentric/allocentric frameworks, we familiarised infants to a single object-centred location presented in six different orientations of the object. Varying the object's orientation across the familiarisation phase is a measure taken to desensitise the infants to the object's coordinates in an egocentric spatial frame of reference (Slater, Morison \& Rose, 1983).

The object was presented in a different orientation on each of the six familairization trials. For all participants the object underwent a fixed order of rotations between trials: Trial 1-2: $225^{\circ}$ anticlockwise, trial 2-3: $180^{\circ}$ clockwise, trial 3-4: $135^{\circ}$ clockwise, trial 4-5: $180^{\circ}$ anticlockwise, trial 5-6: $135^{\circ}$ clockwise. For each infant, the object started in one of two 'starting orientations', and thus the orientation of the object on each trial depended on the starting orientation of the object. The two resulting series of orientations are shown in Figure 3.

--Figure 3 about here--

On each familiarisation trial the same object-centred spatial location was lit up. Thus, the object-centred location shown to each participant was invariant across all familiarisation trials. An example familiarisation phase is shown in Figure 3. In this example the familiarised object-centred location is OCL 2, and the starting orientation of the object is ' 1 '. 
The test phase comprised two trials. In each test trial we presented two Tshaped objects, side by side in the same orientation. Between test trials, both objects remained in the same orientation. On both test trials the familiarised OCL was lit up within one object, and the assigned novel OCL was lit up within the other. This was reversed in the second test trial. Thus, the object which displayed the novel OCL on test trial 1 showed the familiar OCL on test trial 2. The object which displayed the familiar OCL on test trial 1, showed the novel OCL on test trial 2. The order in which the novel/familiar stimuli were presented on left and right objects was systematically varied across subjects.

The orientation of the objects at test was determined by the series of orientations presented during familiarisation. Participants presented with Familiarisation Series 1 during familiarisation were presented with Test Orientation 1, and participants presented with Familiarisation Series 2 were given Test Orientation 2 (see Figure 3). This ensured that the novel and familiar OCLs are presented within a completely novel orientation of the object in the test trials.

Half the infants in both age groups were given novel-familiar contrasts between OCL 1 and OCL 3, and the other half were given novel-familiar contrasts between OCL 2 and OCL 3 (see Figure 1). Successful discrimination between OCLs 1 and 3 requires the representation of a spatial relation to only a single spatial axis of the object (1D comparison). However, the contrast between OCLs 2 and 3 requires more complex two axis spatial reference (2D comparison). Thus, half the infants in each age group were given the 1D comparison and the other half the 2D comparison. Infants receiving a $1 \mathrm{D}$ comparison were either familiarised to OCL 1 , or OCL 3 , and were respectively assigned OCL 3 , or OCL 1 as the novel OCL. Likewise, infants presented with a $2 \mathrm{D}$ comparison were either familiarised to OCL 2 or OCL 3, and 
were respectively assigned OCL 3 or OCL 2 as the novel OCL. A schematic example of a $1 \mathrm{D}$ and a $2 \mathrm{D}$ experimental session is given in Figure 3.

The left-right Order in which the novel stimulus was presented on test trials, Dimension comparison, Novel OCL, and Familiarisation Series were all equally counterbalanced within both age-groups.

\section{Participants}

Forty-one infants took part in this study. The data from sixteen 4-month-olds (of whom 9 were male and 7 female) were included in the analysis. These 16 infants had a mean age of 118.4 days $(\mathrm{SD}=4.0)$. A further seven 4-month-olds were tested, but their data were not included due to fussing (6) or a complete left side bias at test (1). The data of sixteen 8-month-olds (of whom 10 were female, and 6 male) were also included in the analysis. These 16 infants had a mean age of 240.8 days $(\mathrm{SD}=$ 3.9). A further two 8-month-olds were tested but were excluded from the analysis due to fussing (1) or a complete right side bias at test trial (1). The infants who participated in this experiment were selected on the basis of their parents volunteering to take part in the research programme.

\section{Apparatus}

All three limbs of the two T-shaped objects were $12.5 \mathrm{~cm}$ long from the point of intersection, $4 \mathrm{~cm}$ in width, and $4 \mathrm{~cm}$ in depth. Each OCL consisted of five green light emitting diodes (LEDs), fixed inside the T-shapes and concealed (when not illuminated) behind a square window made from diffuse plexiglass. The objects were mounted on a pole extending back in depth from the point of rotation. These poles were also mounted on a flat surface, so that, when rotated, the objects' long axes moved within the frontal plane of an upright infant observer. Everything behind the T-shapes, including the poles, was concealed behind a black screen. Behind the 
concealing screen Experimenter 1 was able to change the orientation of the familiarisation object by rotating the pole discreetly. Experimenter 1 also controlled the lights in the T-shapes from a remote button box. The concealing screen displayed no salient environmental landmark cues.

The infants viewed the object(s) whilst sitting in an upright position on a parent's lap so that their eyes were roughly 60 centimetres from the stimulus. The seating position placed the infants such that during familiarisation the object was at their midlines. The longest axis of the T-shape (top to bottom in Figure 1) presented roughly $22.6^{\circ}$ of visual angle to the infant participants. Thus, if the infant were fixating the point of rotation, the end of each of the limbs extended roughly $11.3^{\circ}$ into the periphery. In the test phase the two object presented together were separated by $30 \mathrm{~cm}$ (14 degrees of visual angle) between their points of rotation. The whole test display subtended 44 degrees of visual angle.

A second experimenter (Experimenter 2) coded the infants' fixations via a discreet infra-red camera placed 35 centimetres below the objects' points of rotation, at the midline. The procedure involved 'accumulated looking' familiarisation and testing (i.e. each trial lasted until the infant had regarded the object for a fixed amount of time), and so we wrote a programme to record accumulated looking, and also to cue the first experimenter when to switch the OCLs on and off. The programme cued Experimenter 1 to turn the OCL on at the beginning of each trial with an audible beep. Once Experimenter 2 had recorded observed and recorded 15 seconds of object directed looking from the infant (using a millisecond timer) the computer produced a second beep to cue Experimenter 1 to turn off the OCL(s) and proceed to the next trial. These beeps were audible to the infants and could obviously provide a cue to the turning on or off of the OCL. However as the beeps were directed solely towards 
the infants' midlines and did not change direction, they could provide no cue to the egocentric location of the OCL, as this varied with respect to the infants' egocentric coordinates throughout familiarisation and test.

\section{$\underline{\text { Procedure }}$}

Before testing began, we asked the parent to try to keep the infant in a constant upright posture, and not to direct the child's attention during the whole procedure. We also asked them to shut their eyes during the stimulus presentation.

To keep the stimuli interesting to the infants, regularly spaced squeaking noises (every 3 seconds) were made from behind the screen during both familiarisation and test trials. There was an interval of approximately 3 seconds between each burst. The location of the squeaking bursts was kept at the infants' midlines throughout in order to avoid any possible side bias in the infants' looking.

The experimental session began once the parent had sat down and turned the infant round to face the familiarisation T-shape. The onset of each trial was signified by a short tone from the timing computer. Each familiarisation trial began with a single location lighting up (the familiarised OCL). When the infant had looked at the T-shape object (the whole object, not just the light) for 15 seconds a second tone sounded and the first experimenter turned the light off. The first experimenter then rotated the T-shape to a new orientation (the rotation of the object was fully visible to the infant), and the next familiarisation trial would then begin (inter-trial interval was set to 4 seconds). For each infant, the light event appeared in the same object-centred location on every familiarisation trial.

Next followed a break in the experimental session of roughly 30 seconds, during which time the infants was moved out of the testing cubicle, and two objects were arranged next to each other in their correct testing orientations. The first test 
trial commenced, with the lights being turned on once the objects were in place, and the infant was settled again for the test phase. It is important to note at this point that the infants do not see the objects being rotated into their novel test orientations. As a consequence they cannot use previous viewer-centred appearances of the light and subsequent rotations of the object to make the discrimination. The discrimination requires that the infants form a representation of the whole object.

The beginning of each test trial was signalled by a short tone from the timing computer. Once the infant had accumulated 15 seconds of total looking to both of the objects, recorded via the millisecond timer buttons, the computer signalled the end of the trial by another short tone, and Experimenter 1 turned the OCL lights off. Between the two test trials both objects remained in full view, without any OCLs lit up. The interval between the test trials was set at 4 seconds.

Because on-line time-keeping is an essential component of this procedure, we examined the reliability between Experimenter 2's on-line looking time scores, and the same experimenter's looking time scores from off-line viewings of video records (intra-observer reliability). This enables us to determine whether Experimenter 2 was consistent in her looking time ratings whilst coding on-line. In order to avoid observer bias, Experimenter 2 was blind to the novelty/familiarity of each object on each trial, whether coding on-line or off-line.

Observer reliability for Experiment 1 was calculated from a sample of 16 test trials of 8 randomly selected infants. The intra-observer reliability between on-line and off-line scores was high (Pearson's $r=0.90$ ).

Results

Familiarisation trials 
Due to the fixed-trial accumulated looking procedure, all infants looked at the object for 15 seconds during each familiarisation trial. Thus, each infant looked at the object + light event for a total of 90 seconds during the familiarisation phase. Table 1 details the length of time it took the infants of both age-groups to reach the familiarisation criterion across three blocks of familiarisation trials. Trial block 1 includes familiarisation trials 1 and 2, trial block 2 includes familiarisation trials 3 and 4, and trial block 3 includes familiarisation trials 5 and 6 . The 4-month-olds took marginally longer than the 8-month-olds in total, and this difference seems to have been most apparent towards the end of the familiarisation phase (in the last two trial blocks). Whilst the 8-month-olds remained relatively constant in their level of interest, the 4-month-olds appear to have habituated to or become fatigued with the stimuli.

--Table 1 about here--

A repeated-measures ANOVA of one within-subjects factor (Trial Block: 1,2 or 3) and one between-subjects factor (Age-Group: 4-month-olds or 8-month-olds) was performed on the length of time infants took to accumulate the required looking criterion of 30 seconds within each familiarisation trial block. This analysis revealed no main effect of Trial Block, but a marginally significant interaction of Trial Block with Age-Group $(\underline{\mathrm{F}}(2,60)=3.1, \mathrm{p}=.051)$. A main effect of Age-Group also approached significance $(\underline{F}(1,30)=3.4, \underline{p}=.076)$. The trends revealed by this analysis suggests a confirmation that the 4-month-olds either habituated or became fatigued more quickly than did the 8-month-olds.

\section{$\underline{\text { Test trials }}$}

The percentage of test trial looking by each infant directed towards the object showing the novel OCL was recorded. Any significant deviation of this score from 
$50 \%$ indicates discrimination of the two stimuli. The mean of this preference across the whole sample was $46.2 \%$, a familiarity preference which was significantly lower than that expected by chance $(\underline{\mathrm{t}}(31)=2.2, \mathrm{p}=.038)$. Table 2 shows the familiarity preference as a function of age and the complexity of the discrimination presented. From this figure it appears that the familiarity preference is mostly due to the 8 month-olds, as the 4-month-olds showed hardly any preference in both dimension groups. In addition, the 8-month-olds demonstrated a reduced preference in the 2dimensional discrimination condition.

--Table 2 about here--

We analysed all groups' test trial percentage novelty preference scores using a repeated measures ANOVA of 1 within-subjects factor (Test Trial: 1 or 2 ) and two between-subjects factors (Age-Group: 4-month-olds or 8-month-olds, and Dimension: 1D or 2D). In an initial analysis of the infants' looking preferences we found a substantial variation between subjects. Variation in novelty preference scores is thought to be linked to individual differences in the speed at which infants habituate to the familiarisation stimuli (Cohen, 1969). It seems reasonable to assume that the infants who take the longest time to reach the 90 second familiarisation criterion have habituated earlier than those who reached criterion rapidly. A prediction which follows is that the infants who take longer to reach criterion will be more likely to show novelty preferences than those who reach criterion rapidly. Given this individual variability, we also included the total time taken to reach criterion during familiarisation as a covariate in our analysis (familiarisation duration). This analysis revealed a significant main effect of Age-Group $(\underline{\mathrm{F}}(1,24)=8.2, \underline{\mathrm{p}}=.009)$, and a significant covariation of novelty preference with familiarisation duration $(\underline{F}(1,24)=11.6, \quad p=.002)$. There was also a significant interaction between 
familiarisation duration age-group $(\underline{\mathrm{F}}(1,24)=7.3, \underline{\mathrm{p}}=.012)$. There were no main effects or interactions of Test Trial or Dimension.

The main effect of Age-Group confirms that the 8-month-olds demonstrated a greater average familiarity preference than the 4-month-olds. The significant covariation of novelty preference with familiarisation duration (time taken to reach criterion) indicates that there is a relation between the time an individual infant takes to familiarise and the preference that they demonstrate at the test trial. This is consistent with previous research on habituation showing that depth of habituation is an important determinant of the degree of novelty preference observed (Hunter \& Ames, 1988; Fantz, 1964; Lipsitt, 2002, Sirois \& Mareschal, 2002). The interaction of this covariation with age-group indicates that the way in which familiarisation duration affects novelty preference varies between age-groups. We explored this interaction further by computing the correlation between familiarisation duration and novelty preference scores within each age-group.

\section{--Figure 4 about here--}

Figure 4 demonstrates that the correlation between familiarisation duration and novelty preference is driven by the behaviour of the 8-month-old infants. The significant positive correlation in this age-group shows that the 8-month-olds who took less time to familiarise to the OCL across the six orientations of the familiarisation phase were more likely to demonstrate a strong preference for the familiar, whereas those who took longer to familiarise directed more of their attention towards the novel OCL. Length of familiarisation is an indication of the degree to which an infant has habituated to the familiarised stimulus. Those who took longer to complete the familiarisation phase here (those who have habituated earliest) have shown a reduced preference for the familiar at test (and thus a greater novelty 
preference) than those who completed the familiarisation phase more quickly (those who have habituated latest or not at all). In contrast, the 4-month-olds did not differ significantly from $50 \%$, whatever the duration of familiarisation.

Importantly, the consistent relation between preference on test trials, and familiarisation duration demonstrates that 8-month-olds can encode and discriminate between object-centred spatial locations. Indeed this relation holds up for both the simple 1D discrimination $(\underline{\mathrm{r}}=.82, \underline{\mathrm{N}}=8, \mathrm{p}=.014)$ and the complex $2 \mathrm{D}$ discrimination $(\underline{\mathrm{r}}=.85, \underline{\mathrm{N}}=8, \underline{\mathrm{p}}=.007)$.

\section{Discussion}

These results clearly indicate that 8 -month-old infants are able to make objectcentred spatial discriminations despite changes in object orientation, and furthermore that they are able to do this even when the discrimination pairs are only differentiated by reference to multiple axes of the object-centred frame of reference. In contrast, the 4-month-old infants whom we tested demonstrated no such ability even when the discrimination pairs were differentiated by simple reference to one object-centred spatial axis.

There are two reasons to be cautious before drawing strong conclusions from the null finding with the younger age-group. Firstly, we have previously found that 4month-olds could make simple 1D discriminations under different experimental conditions (Bremner et al, in 2006). Using the same T-shaped stimuli as those employed here, we found that 4-month-olds would demonstrate a post-familiarisation preference for the novel OCL (in a 1D pair), but when tested using a shorter familiarisation period (10 seconds of accumulated looking per trial, as opposed to the 15 used here) and with successive (rather than simultaneous) novel and familiar test trial presentation. 
Secondly, we cannot conclude that differences in looking behaviour between two age-groups, given a constant experimental procedure, is necessarily driven by an underlying change in perceptual/cognitive competence. Looking behaviour is an indirect measure of perceptual/cognitive competence, and as such it is always possible that any age-group differences reflect a developmental change in the looking response behaviour itself, rather than the cognitive/perceptual skill under investigation. It could be that the 4-month-olds have shown no discrimination here because of a lesser tendency in this age group to show preferences for familiar stimuli, rather than an inability to make the spatial discrimination. Indeed, age is one of the three factors implicated in Hunter \& Ames's (1988) model of novelty and familiarity preferences in infancy. However, Hunter \& Ames's model and its supporting experimental evidence (Wetherford \& Cohen, 1973) suggests, in contrast to the pattern shown here, that 4-month-olds would show a greater post familiarisation familiarity preference than 8-month-olds, as younger age-groups are thought to take longer to encode visual information before showing an orienting preference towards novel stimuli.

Thus, it seems more likely that the 4-month-olds may have demonstrated no consistent preference due to the onset of fatigue before the beginning of the test phase. In fact there is a suggestion in the familiarisation data that this was indeed the case. Whereas most studies of infant habituation would suggest that the younger agegroup would habituate more slowly (and thus complete the familiarisation test phase faster) than the older age-group (Slater, 1995; Hunter \& Ames, 1988), Table 1 shows that the 4-month-olds' looking durations were actually higher than those of the 8month-olds in the last two familiarisation trial blocks. This age-group difference was reflected in our analysis of familiarisation duration; the interaction of familiarisation trial block with age-group approached significance. As argued above it seems 
unlikely that the 4-month-olds would habituate more quickly than the 8-month-olds. Thus, it seems plausible that this trend is due to the earlier onset of fatigue in the 4month-old group.

Thus, Experiment 2a examines whether 4-month-olds might show evidence of object-centred spatial discrimination when the familiarisation criterion is reduced in order to avoid fatigue.

\section{Experiment 2a}

Experiment 2a presented a group of 4-month-old infants with the same fixed trial familiarisation procedure as that used in Experiment 1, but with the familiarisation criterion set this time at 10 seconds of cumulative looking per trial rather than 15 seconds per trial as was used in Experiment 1. With this criterion the infants were familiarised to 60 seconds of accumulated looking across the whole familiarisation phase, rather than the 90 seconds that the infants in Experiment 1 were exposed to. In addition, because we have already demonstrated elsewhere (Bremner et al, 2006) that 4-month-olds are able to make simple 1D discriminations of objectcentred location, this experiment tested the more complex 2D discriminations only.

$$
\text { Method }
$$

\section{Design}

The design of Experiment 2a was the same as that of Experiment 1 with the following exceptions. We assigned each infant with a comparison between novel and familiar locations which required a 2D discrimination. All infants were presented with an OCL comparison between OCLs 2 and 3. The OCL that was assigned as 'novel' was varied between participants. Thus infants were either familiarised to OCL 2, or OCL 3, and were thus respectively assigned OCL 3 or OCL 2 as the novel OCL.

\section{Participants}


Eight infants participated in Experiment 2a (4 female, and 4 male). Their mean age was 123 days $(\mathrm{SD}=7.9)$. A further three babies were tested but their data were excluded due to fussiness (2), and experimenter error (1).

\section{Apparatus and procedure}

Apparatus was identical to that used in Experiment 1. The procedure was the same apart from two differences. In the familiarisation phase of this experiment we only required that the infants accumulate 10 seconds of looking on each familiarisation trial. The two test trials both also lasted for 10 seconds of accumulated looking. The second difference between this procedure and that of Experiment 1 was the absence of the squeaking bursts behind the screen during familiarisation and test trials. While the bursts could not have had any differential effect on novelty/familiarity preferences at test trials in Experiment 1, it is possible that they may have affected the overall level of arousal and thus the level and quality of encoding of the visual stimuli.

\section{Results}

\section{$\underline{\text { Familiarisation trials }}$}

All infants looked at the object for 10 seconds during each familiarisation trial. Thus, each looked at the object + light event for a total of 60 seconds during the familiarisation phase. On average it took the infants 106.0 seconds $(\mathrm{SE}=15.3)$ to accumulate 60 seconds of looking within the familiarisation period. Within familiarisation trial blocks 1,2 , and 3 the infants took 38.4 seconds $(\mathrm{SE}=9.5), 32.2$ seconds $(\mathrm{SE}=3.8)$, and 35.4 seconds $(\mathrm{SE}=3.6)$ respectively to accumulate the 20 seconds of looking required within each block. We conducted a one-way repeated measures ANOVA on the duration of the familiarization trials across the three familiarisation trial blocks. This revealed no effect of Trial Block $(\underline{F}(2,14)=0.5$, n.s. $)$. 
--Table 3 about here--

\section{$\underline{\text { Test trials }}$}

Seven of the eight infants tested showed an overall preference for the object in which the familiar OCL was highlighted. On average infants directed 37.2\% (SE = 3.3) of their total object-directed looking towards the object in which the novel OCL was highlighted (see Table 3). These data were subjected to a repeated measures ANOVA with one within-subject variable (Test Trial: 1 or 2), and one covariate (duration of the familiarisation period in seconds). There were no main effects of Test Trial $(\underline{\mathrm{F}}(1,6)=0.013$, n.s. $)$ or familiarisation duration $(\mathrm{F}(1,6)=0.16$, n.s. $)$. Neither did we find an interaction between these two factors $(\underline{F}(1,6)=0.09$, n.s. $)$. We next conducted a one-sample t-test in order to determine whether the infants' percentage preferences for the novel OCL differed significantly from chance (50\%). As there was no effect of test trial, we used the infants' total preference across both trials in this analysis. The $37.2 \%$ score (a familiarity preference) was found to be significant $(\underline{t}(7)=3.9, \underline{p}=.006)$.

\section{Discussion}

The infants tested in this study demonstrated a consistent test trial preference for the object in which the familiar object-centred location was lit up. With the current experimental design this result can be interpreted in two ways.

Firstly, it is possible that the preference for the familiar OCL represents a visual preference for a novel location with respect to spatial coordinates that are not solely defined relative to the object's frame of reference. In the familiarisation phase, a single object-centred location was presented in six different environmental and egocentric locations, by virtue of the reorientation of the object between each of six familiarisation trials. In the test phase, two objects were presented to either side of 
the location where the familiarisation object had appeared in the familiarisation phase. While both test objects were presented in orientations such that both of the test OCLs were in new locations with respect to absolute environmental and body-centred spatial coordinates, it is still possible that the infants could have been coding location in environmental and egocentric space with respect to the rough position of the objects within such frames of reference (what we will refer to as a 'landmark' spatial code).

Because of the particular set of orientations that we used during the familiarisation phase of Experiment 2a, the familiar OCL appeared to one side of (either above or below) the central point of rotation of the object two more times than the other. On test trials the objects were oriented such that the novel OCL was in a familiar location with respect to this landmarked spatial framework, and the familiar OCL in a novel location with respect to this framework. This point of design was initially included so that a preference for the novel OCL could not be explained by its novelty with respect to non object-centred spatial coordinates. However, a preference for the familiar OCL, as was found here could represent a preference for a novel location in the egocentric field with respect to the object (a novel landmarked location).

The distribution of landmark spatial locations that the light occupies during the familiarisation phase is shown in Figure 4, where the light appears twice on the object-defined horizontal axis, once below it and three times above it. In both subsequent test trials the familiar OCL appears directly below the object's point of rotation. Thus, in this particular condition it is possible that the infants developed and familiarised to a generalised representation of 'above the object' during the 
familiarisation phase, and subsequently preferred the familiar OCL in the test trials due to its novel location with respect to the landmark spatial framework.

It should be noted at this stage that this particular interpretation cannot account for the familiarity preference found in the 8-month-old group in Experiment 1. There, the significant correlation between the novelty/familiarity preference at test with the total duration of the familiarisation phase indicated that those individuals who took longer to familiarise (who habituated faster) directed less looking towards the familiar OCL. This finding suggests that the 8-month-olds were responding to the object-centred location and not location with respect to any landmark spatial framework.

The second possible interpretation of the results of Experiment 2a, and the one more pertinent to our research question, is that the infants looked longer at the familiar OCL stimuli due to a preference for the familiar OCL over the novel OCL. This would indicate that 4-month-old infants are able to make discriminations of object-centred location that require representations of location in relation to two spatial axes of the object-centred framework; '2D OCL discriminations'. Experiment $2 \mathrm{~b}$ tests these interpretations.

\section{Experiment $2 b$}

The aim of Experiment $2 \mathrm{~b}$ is to distinguish between the two explanations of the visual preference offered here, by equating the two test stimuli on the basis of their novelty with respect to environmental and egocentric spatial coordinates. In order to do this we conducted a further fixed-trial familiarisation experiment in which novel and familiar OCL stimuli are equated for their novelty with respect to environmental and egocentric spatial coordinates. It is possible to do this by changing the sequence of rotations used in the familiarisation phase. Thus, the sequence of 
rotations that we decided to employ in the familiarisation phase of this experiment presents the familiar OCL in a balanced distribution of egocentric and environmental locations. The test phase presents familiar and novel OCLs in locations that are equally familiar or novel with respect to environmental and egocentric space relative to the object landmarks.

If the preference found in Experiment 2a is due to the novelty of the OCL in relation to environmental/egocentric reference with respect to the object landmarks, then we would predict there to be no preference in the current experiment. However, if the preference found in Experiment 2a was due to the familiarity of the familiarised OCL then we would predict a similar familiarity preference in the current experiment.

$$
\text { Method }
$$

\section{$\underline{\text { Design }}$}

The design was the same as that used in Experiment $2 \mathrm{a}$ except that in the familiarisation phase of Experiment $2 \mathrm{~b}$ the object underwent the following fixed order of rotations between trials: trial 1-2: $180^{\circ}$ anticlockwise, trial 2-3: $135^{\circ}$ clockwise, trial 3-4: $180^{\circ}$ clockwise, trial 4-5: $225^{\circ}$ anticlockwise, trial 5-6: $180^{\circ}$ clockwise.

Like Experiments 1 and 2a, just two starting orientations were used. The starting orientations that we used here are different to those used previously, resulting in two possible sequences of orientations during the familiarisation phase. These are shown in Figure 5.

--Figure 5 about here--

The orientation of the objects in the test phase means that the novel OCL light (in this case OCL 2) is in a location 'below the object' landmarked location that was not occupied by the familiarisation OCL on any of the familiarisation trials. The same is true of the familiar OCL (in this case OCL 3) which occupies an 'above the 
object' landmarked location which is also novel. Also, the number of times that the familiar OCL appears above and below the object-landmarked horizontal axis during the familiarisation phase is balanced (twice above, twice below, twice coincident with).

This particular fixed set of rotations thus equates novel and familiar OCLs at test trial on the basis of the novelty/familiarity of their locations with respect to object-landmarked allocentric/egocentric space.

\section{$\underline{\text { Participants }}$}

Eight infants participated in Experiment $2 \mathrm{~b}$ (2 male, and 6 female). Their mean age was 122 days $(\mathrm{SD}=6.1)$. One other infant was tested but not included in the analysis due to fussy behaviour.

Apparatus and procedure

Apparatus and procedure were identical to those used in Experiment 2a. Observer reliability for Experiments $2 \mathrm{a}$ and $2 \mathrm{~b}$ was calculated from a sample of 18 test trials of 9 randomly selected infants. The intra-observer reliability between online and off-line scores was high (Pearson's $r=0.88$ ).

Results

\section{Familiarisation trials}

All infants looked at the object for 10 seconds during each familiarisation trial. Thus, each looked at the object + light event for a total of 60 seconds during the familiarisation phase. On average it took the infants 138.3 seconds $(\mathrm{SE}=12.9)$ to accumulate 60 seconds of looking within the familiarisation period. Within familiarisation trial blocks 1,2 , and 3 the infants took 47.9 seconds ( $\mathrm{SE}=7.8), 47.0$ seconds $(\mathrm{SE}=6.7)$, and 43.4 seconds $(\mathrm{SE}=10.6)$ respectively to accumulate the 20 seconds of looking required within each block. We conducted a one-way repeated 
measures ANOVA on the duration of the familiarization trials across the three familiarisation trial blocks. This revealed no effect of Trial Block $(\underline{F}(2,14)=0.7$, n.s. $)$

\section{$\underline{\text { Test trials }}$}

All eight infants tested showed an overall preference for the object in which the familiar OCL was highlighted. On average these infants directed $38.5 \%$ (SE $=$ 3.6) of their total object-directed looking towards the object in which the novel OCL was highlighted (see Table 3). These data were subjected to a repeated-measures ANOVA with one within-subject variable (Test Trial: 1 or 2), and one covariate (familiarisation duration (seconds)). There were no main effects of Test Trial $(\underline{F}(1,6)=3.1$, n.s. $)$ or familiarisation duration $(F(1,6)=2.7$, n.s. $)$. Neither did we find an interaction between these two factors $(\underline{F}(1,6)=2.6$, n.s.). We next conducted a onesample t-test in order to determine whether the infants' percentage preferences for the novel OCL differed significantly from chance $(50 \%)$. As there was no effect of test trial, the infants' total preference score across both trials was used in this analysis. The $38.5 \%$ score (a familiarity preference) was found to be significant $(\underline{t}(7)=3.2$, $\mathrm{p}=.015)$.

To assess the effect of changing the orientation series used during familiarisation we ran a repeated-measures ANOVA on all of the infants' test trial preferences across Experiments $2 \mathrm{a}$ and $2 \mathrm{~b}$. The ANOVA included one withinsubjects factor (Test Trial: 1 or 2), one between subjects factor (Experiment: 2a or 2b), and one covariate (familiarisation duration). We found no main effect of Test Trial $(\underline{\mathrm{F}}(1,12)=2.5$, n.s. $)$, Experiment $(\underline{\mathrm{F}}(1,12)=2.4$, n.s. $)$, or familiarisation duration $(\underline{F}(1,12)=0.9$, n.s. $)$. There were no significant interactions between any of these factors (the largest interaction effect was between test trial and experiment $(\underline{F}(1,12)=2.9$, n.s. $) . \quad$ A one-sample t-test showed that the preference for the familiar 
OCL (across both test trials) was significant in this combined analysis $(\underline{\mathrm{t}}(15)=5.2$, $\mathrm{p}<0.001)$.

\section{Discussion}

The 4-month-olds tested here showed a consistent preference for the stimulus in which the familiar OCL was lit up. In this experiment, the novel and familiar OCLs were equated for their novelty with respect to environmental/egocentric landmark coordinates. There were no significant differences in behaviour between Experiments $2 \mathrm{a}$ and $2 \mathrm{~b}$, and consequently we interpret the findings of both experiments as a preference for the familiar object-centred location.

The familiarity preference indicates that the infants were able to make a perceptual discrimination between the test stimuli on the basis of their novelty/familiarity with respect to the familiarisation stimuli. In the current context this ability indicates that 4-month-old infants are able to discriminate between two object-centred spatial locations, regardless of changes in the object's orientation with respect to egocentric/environmental spatial coordinates. Furthermore, the novelfamiliar OCL pairs presented in Experiments $2 \mathrm{a}$ and $2 \mathrm{~b}$ were only differentiable by coordinated reference to two axes of the object-centred spatial frame of reference. We can conclude that 4-month-old infants can make this complex 2D object-centred spatial discrimination.

\section{General Discussion}

Following familiarisation to a single object-centred location presented in six different orientations of the object, the 8-month-olds tested in Experiment 1, and the 4-month-old infants tested in Experiments $2 \mathrm{a}$ and $2 \mathrm{~b}$ demonstrated a preference for the familiar object-centred spatial configuration over a novel one, despite both configurations being presented in novel orientations and locations with respect to the 
infants' egocentric axes. This result confirms and extends Bremner et al's (2006) finding that 4-month-old infants are able to notice changes in feature location relative to an object-centred frame of reference, independently of egocentric and allocentric frames of reference. Moreover, this ability is also available at eight months of age.

These findings have a crucial bearing on the nature of object recognition in infancy and adulthood. Recognition of objects on the basis of structural configuration alone is of course only one of the strategies available for recognising objects across changes in orientation. Under ecological conditions there is generally much more featural information specifying the identity of parts, providing an adequate input to part-based recognition, without recourse to structural descriptions. However, object categorisations at what has been termed the subordinate level (Biederman, Subramaniam, Bar, Kalocsai \& Fiser, 1999) are much less rich in part identity distinctions, and can thus benefit more from structural (object-centred spatial) descriptions. Indeed, there is general agreement among almost all theories of object recognition that some level of object-centred description is formed in nearly all acts of recognition (Mozer, 2002; Tarr \& Pinker, 1990; Biederman, 1987).

Evidence that infants are able to represent spatial layout relative to one axis of an object-centred framework strengthens the supposition that object-centred descriptions play an important part in human object-recognition. Furthermore, the results here also show that 4 - and 8 -month-olds are also able to make discriminations of object-centred spatial configurations that require localisation with respect to two coordinated axes (or dimensions) of the object's framework. Tarr and Pinker (1989, 1990) present evidence indicating that as adults we achieve these more complex discriminations by imagining the transformation of a mental image of the object in order to compare it against a stored egocentric object representation (Shepard \& 
Metzler, 1971; Tarr \& Pinker, 1989; 1990)². So what are we to make of the infants' abilities? Are we to conclude that infants of 4 months, like adults, are able to rotate mental images of object shapes in order to match them to stored egocentric representations?

\section{Mental rotation in infancy}

Rochat \& Hespos (1996; Hespos \& Rochat, 1997) have proposed the existence of mental rotation abilities in infancy. Using a 'violation of expectation' looking paradigm, they undertook a series of experiments in which 4- 6- and 8-month-old infants were tested at their ability to track and anticipate the final orientation of an object following dynamic displacements and rotations which were partly obscured. In order to anticipate the correct resting orientation of the object infants had to use information about the rotatory and/or translatory movement of the object before it became obscured. All age-groups demonstrated looked longer when the object was revealed to have come to rest in an orientation that was inconsistent with its prior trajectory ${ }^{3}$. It is tempting to use Rochat and Hespos's findings as a corroboration of our own, positing a mental rotation faculty in early infancy. However, there are reasons to question whether their experimental procedure tests mental imagery. It can be argued that the rich dynamic information provided before the occlusion of the object in their experiments could support a prediction of orientation through interpolation, side-stepping the need to invoke dynamic imagery. There is certainly plenty of evidence to suggest that young infants are very capable of predicting the trajectories of moving objects across spatial and temporal gaps (Bower, Broughton and Moore, 1971; Johnson et al, 2003), and there seems no reason not to extend this ability to rotatory trajectories. 
Indeed, is argued by some researchers that dynamic visual information is of primary importance in early object recognition (e.g. Kellman, 1984). At the test phase in the experiments reported in the current paper, the objects were presented in a novel and stationary orientation. It thus seems that even at 4 months infants were able to recognise the spatial configuration of our objects without this information being provided in the context of a dynamic perceptual event ${ }^{4}$.

Most research into the development of mental rotation has suggested that the mental operations required for mental transformation of egocentric spatial configurations emerge in middle childhood, rather than early infancy (Huttenlocher \& Presson, 1973; Harris \& Bassett, 1976; Newcombe \& Huttenlocher, 2000; Olson \& Bialystok, 1986; Piaget \& Inhelder, 1956; Scholnick, Fein \& Campbell, 1990). By way of illustration, Piaget and Inhelder's (1948/1956) famous 'Three Mountains' task uncovered a sequence of development in which children become gradually more sophisticated at reasoning about the effect of viewpoint on the appearance of a visual scene. When asked to choose a picture (from a variety of perspectives), which most accurately portrayed the view of the scene from the opposite side, 5-7 year olds typically managed some kind of transformation (front/back or left/right), but it was not until 8 years (at Piaget's stage of concrete operations) that all children managed to correctly coordinate transformations of both of these dimensions to consistently identify the correct alternative viewpoint.

Due to the uncertain representational basis for competence demonstrated in Rochat \& Hespos's studies, and the power of evidence in favour of the development of mental rotation abilites in later childhood it does not seem safe to assume that an early ability at mental rotation underlies the $2 \mathrm{D}$ object recognition skills demonstrated by the 4- and 8-month-olds in the series of experiments reported here. However, 
there are other ways of achieving this competence than through mental rotation. It is possible that the precocious abilities demonstrated here arise as a result of a completely different approach to object encoding in early infancy.

As already related, adults are able to remember the configuration of an object with respect to a single axis of the object-centred spatial framework. However, they appear unable to use an object-centred code to distinguish spatial configurations which are only differentiated with respect to more than one axis of the object; in this case they use mental rotation in order to match such configurations against stored egocentric representations (Tarr \& Pinker, 1990). However it is not a computationally intractable problem to form an object-centred representation that defines a configuration relative to 2 axes. An alternative interpretation of the early competence demonstrated by the 4- and 8-month-olds is that they may actually be able to form more complex externally referenced spatial representations than adults and young children. This suggestion may not be as unreasonable as it at first seems; it may actually be more behaviourally adaptive for young infants to use external spatial reference. We will unpack this line of reasoning below.

\section{Competence with external frames of reference in infancy}

By comparison to young children and adults, infants between four and eight months of age have much less need to attend to the egocentric frameworks required for establishing an active role in their environment. At this stage they are only just beginning to develop object manipulation skills, and certainly very few are actively locomoting (Bayley, 1969; Bertenthal, Campos \& Barrett, 1984; Campos, Anderson, Barbu-Roth, Hubbard, Hertenstein \& Witherington, 2000; Hofsten \& Fazel-Zandy, 1984; Hofsten \& Rönnqvist, 1988). If we take into account that infants have less need to use egocentric reference in relation to action, and further acknowledge the inherent 
unreliability of egocentric reference for encoding visual information in a constantly transforming environment, it no longer seems implausible that young infants might find it more efficient to represent visual location in relation to external, rather than egocentric, spatial frameworks. The logical extension of this argument is that we may actually develop more towards egocentric spatial coding as we become older and take a more active involvement in our surroundings. A very tentative suggestion may thus be that young infants may be able to encode relatively complex (2D) external spatial configurations, but become less prone to use this ability as they become more actively and egocentrically involved in their object representations later in development.

\section{$\underline{\text { Infants' visual preferences for novel and familiar }}$}

It is also of interest to ask why the infants tested here showed predominant preferences for the familiar object-centred configuration at test. Whilst the direction of infant preference is not of key relevance to our hypotheses concerning the presence of a discriminative or recognition ability, it is thought to reflect the quality of representation of the novel and familiar stimuli. In their model of infant preference for novel or familiar, Hunter and Ames (1988) propose that infants demonstrate familiarity preference when they have still not completed encoding of the familiar stimulus to an acceptable level of certainty, given a specific discrimination. Three factors are offered as affecting the demonstration of familiarity or novelty preferences: age (older children attain an acceptable representation more quickly), duration of familiarisation (longer familiarisation is more likely to cross the criterion of acceptability), and difficulty of discrimination (more difficult discriminations require a higher criterion of representational quality).

There are two findings from the current experiments which seem important to discuss in relation to Hunter and Ames's model of novelty/familiarity preference. 
Firstly, in a previous experiment using these stimuli, we (Bremner, Bryant \& Mareschal, 2006) found a significant preference for a novel object-centred location, given a 1D discrimination pair at 4 months. Given this previous finding it is important to justify why we uncovered a familiarity for this same discrimination in experiment, in an older age-group. Hunter and Ames's model would predict a shift further towards novelty in an older age-group. However, there is one important difference between the procedures of our experiments. In the current experiments we paused for around 30 seconds between familiarisation and test phases in order to introduce, whereas Bremner et al moved straight into the test phase with no break. It seems likely that the difference in preference is due to time-related deterioration of, and the interference of extraneous stimuli with, the representation of the familiar stimulus, making the task of comparing it with the novel stimulus more demanding.

Secondly it is interesting to discuss the reasons for finding a relation between the 8-month-old individuals' looking behaviour during the familiarisation phase and the strength/direction of their preference at test in Experiment 1. The 8-month-olds who took longest to reach the familiarisation criterion (90 seconds of accumulated looking) (those who looked away most during the familiarisation phase), demonstrated preference score which were shifted more towards the novel than the infants who accumulated the criterion quickly (those who looked away least during familiarisation). In accordance with Hunter and Ames's (1988) model, this seems to indicate that there was a spread of individual differences in the amount of attention that infants required to familiarise sufficiently enough to show a novelty preference at the test phase. Colombo, Freeseman, Coldren and Frick (1995) have suggested that infants who look away more (those who show shorter look fixations) exhibit a more adult-like attentional profile, giving priority to global rather than local features of the 
visual stimulus before its local features (Navon, 1977). Indeed, Stoecker, Colombo, Frick and Ryther (1998) have found that infants who show shorter fixations are more likely to show a novelty preference when given a post-familiarisation discrimination between symmetrical and asymmetrical stimuli. This would seem to suggest that in our experiments, infants who exhibited looking behaviour typical of more global attention were at an advantage for encoding the stimuli that we presented.

The bestowal of an advantage on object processing by a more global pattern of attention may hint at the underlying representations that the infants formed of the familiarized stimulus. In our discussion of the representations underlying the infants' ability to make the 2D discrimination, we posited two explanations. The first was that the infants (like adults) solved the problem using mental rotation, a solution based on egocentric representations of the stimuli. The second was that the infants may have solved the problem by reference to the spatial layout of the external object-centred frame of reference. It is likely that egocentric encoding is at an advantage when a limited spread of attention reduces variation of the stimulus with respect to retinal coordinates, whereas the object-centred frame of reference (which does not vary across eye-movements) may be emphasized by the variation produced by the eyemovements involved in a more global attentional style. This explanation would favour an interpretation of infant competence in terms of their ability to reference the external object-centred spatial framework

\section{$\underline{\text { Summary and conclusions }}$}

We have shown that 4- and 8-month-old infants are able to make discriminations between object-centred spatial configurations that are only differentiated with respect to two axes of the object's framework. The style of attention demonstrated by the 8-month-olds who showed more efficient encoding of 
the object indicates that the infants used external (not egocentric) spatial reference to make this discrimination. These results and our interpretation stand in contrast with Piaget's constructionist account of the development of spatial representation (Piaget, 1937/1954; Piaget \& Inhelder, 1956). Piaget proposed that infants' spatial representations were initially restricted to egocentric coordinates, with more independent spatial reference developing from active exploration of the environment. Nonetheless we are not alone in suggesting that young infants are able to use external spatial coding. Research reported by Kaufman and colleagues (Kaufman, 1998; Kaufman \& Needham, 1999) demonstrates that at four and six months of age, infants are able to represent the location of an object relative to environmental coordinates, despite variance in its relation to the egocentric spatial frame of reference. Our evidence, and that of Kaufman and colleagues, presents a strong challenge to Piaget's egocentrism hypothesis, and shows that even at only four months of age we can form an objective representation of visual space. 


\section{References}

Bayley, N. (1969). Bayley Scales of Infant Development. Psychological Corporation: New York, NY.

Bertenthal, B. I., Campos, J. J., \& Barrett, K. C. (1984). Self-produced locomotion: an organiser of emotional, cognitive and social development in infancy. In R. Emde \& R. Harnon (Eds.), Continuities and discontinuities in development. Plenum: New York, NY.

Biederman, I. (1987). Recognition-by-components: a theory of human image understanding. Psychological Review, 94, 115-147.

Biederman, I., Subramaniam, S., Bar, M., Kalocsai, P., \& Fiser, J. (1999).

Subordinate-level object classification re-examined. Psychological Research, 62, 131-153.

Bower, T.G.R., Broughton, J., \& Moore, M.K. (1971). Development of the object concept as manifested in changes in the tracking behaviour of infants between 7 and 20 weeks of age. Journal of Experimental Child Psychology, 13, 182193.

Bremner, A.J., Bryant, P.E., \& Mareschal, D. (2006). Object-centred spatial reference in 4-month-old infants. Infant Behavior and Development, 29, 1-10.

Campos, J. J., Anderson, D. I., Barbu-Roth, M. A., Hubbard, E. M., Hertenstein, M. J., \& Witherington, D. (2000). Travel broadens the mind. Infancy, 1, 149220.

Cohen, L. B. (1969). Observing responses, visual preferences, and habituation to visual stimuli in infants. Journal of Experimental Child Psychology, 7, 419433. 
Colombo, J., Freeseman, L. J., Coldren, J. T., \& Frick, J. E. (1995). Individual differences in infant visual fixation: Dominance of global and local stimulus properties. Cognitive Development, 10, 271-285.

Harris, P. L., \& Bassett, E. (1976). Reconstruction from the mental image. Journal of Experimental Child Psychology, 21, 514-523.

Hespos, S. J., \& Rochat, P. (1997). Dynamic mental representation in infancy. Cognition, 64, 153-188.

Hofsten, C. von, \& Fazel-Zandy, S. (1984). Development of visually guided hand orientation in reaching. Journal of Experimental Child Psychology, 38, 208219.

Hofsten, C. von, \& Rönnqvist, L. (1988). Preparation for grasping an object: a developmental study. Journal of Experimental Psychology: Human Perception and Performance, 14, 610-621.

Hummel, J. E. (2000). Where view-based theories break down: the role of structure in human shape perception. In E. Dietrich \& A. B. Markman (Eds.), Cogntive Dynamics: Conceptual Change in Humans and Machines. Erlbaum: Mahwah, NJ.

Hummel, J.E. (2001). Complementary solutions to the binding problem in vision: Implications for shape perception and object recognition. Visual Cognition, 8, 489-517.

Hunter, M. A., \& Ames, E. W. (1988). A multifactor model of infant preferneces for novel and familiar stimuli. In C. Rovee-Collier and L. P. Lipsitt (Eds.), Advances in Infancy Research, Vol. 5. Ablex Publishing: Westport, CT.

Huttenlocher, J., \& Presson, C. C. (1973). Mental rotation and the perspective problem. Cognitive Psychology, 4, 277-299. 
Johnson, M. H., \& Gilmore, R. O. (1998). Object-centred attention in 8-month-old infants. Developmental Science, 1, 221-225.

Johnson, S. P., Bremner, J. G., Slater, A. M., Mason, U. C., Foster, K., \& Cheshire, A. (2003). Infants' perception of object trajectories. Child Development, 74, 94-108.

Kaufman, J. (1998). The Development of Spatial Thinking and Action in Early Infancy. Unpublished Doctoral Thesis, Duke University.

Kaufman, J. and Needham, A. (1999). Objective spatial coding by 6.5-month-old infants in a visual dishabituation task. Developmental Science, 2, 432-441.

Kellman, P.J. (1984). Perception of three-dimensional form by human infants. Perception \& Psychophysics, 36, 353-358.

Marr, D. (1980). Vision. Freeman: New York, NY.

Marr, D., \& Nishihara, H. K. (1978). Representation and recognition of the spatial organisation of three-dimensional structure. Proceedings of the Royal Society of London, Series B (Biological Sciences), 200, 269-294.

Mozer, M. C. (2002). Frames of reference in unilateral neglect and visual perception: a computational perspective. Psychological Review, 109, 156185.

Navon, D. (1977). Forest before trees: The precedence of global features in visual perception. Cognitive Psychology, 9, 353-383.

Newcombe, N. S., Huttenlocher, J. (2000). Making Space: The development of spatial representation and reasoning. MIT Press: Cambridge, MA.

Olson, D. R., \& Bialystok, E. (1983). Spatial Cognition: The structure and development of mental representations of spatial relations. Lawrence Erlbaum Associates: Hilldale, NJ. 
Piaget, J. (1954). The Construction of Reality in the Child. Routledge \& KeganPaul: London, UK.. (Originally published in French in 1937).

Piaget, J., \& Inhelder, B. (1956). The Understanding of Space in the Child. Routledge \& Kegan-Paul: London, UK., (Originally published in French in 1948).

Pylyshyn, Z. W. (1981). The imagery debate: Analogue media versus tacit knowledge. Psychological Review, 88, 16-45.

Quinn, P. C., Slater, A. M., Brown, E., \& Hayes, R. A. (2001). Developmental change in form categorisation in early infancy. British Journal of Developmental Psychology, 19, 207-218.

Rochat, P., \& Hespos, S. J. (1996). Tracking and anticipation of invisible spatial transformations by 4- to 8-month-old infants. Cognitive Development, 11, 317.

Scholnick, E. K., Fein, G. G., \& Campbell, P. F. (1990). Changing predictors of map use in wayfinding. Developmental Psychology, 26, 188-193.

Shepard, R. N., \& Metzler, J. (1971). Mental rotation of three-dimensional objects. Science, 171, 701-703.

Sirois, S., \& Mareschal, D. (2002). Computational approaches to infant habituation. Trends in Cognitive Sciences, 6, 293-298

Slater, A. M. (1995). Visual perception and memory at birth. In C. Rovee-Collier, and L. P. Lipsitt (Eds.), Advances in infancy research (Vol. 9). Ablex: Norwood NJ.

Slater, A. M., Mattock, A., \& Brown, E. (1990). Size constancy at birth: newborn infants' responses to retinal and real size. Journal of Experimental Child Psychology, 49, 314-322. 
Slater, A. M., \& Morison, V. (1985). Shape constancy and slant perception at birth. Perception, 14, 337-344.

Stoecker, J. J, Colombo, J., \& Frick, J. E. \& Ryther, J. S. (1998). Long- and shortlooking infants' recognition of symmetrical and asymmetrical visual forms. Journal of Experimental Child Psychology, 71, 63-78.

Tarr, M. J. (1999). News on views: pandemonium revisited. Nature Neuroscience, 2, 932-935.

Tarr, M. J., \& Bulthoff, H. H. (1998). Image-based object recognition in man, monkey, and machine. In M. J. Tarr and H. H. Bulthoff (Eds.), Object recognition in man, monkey, and machine. MIT Press : Cambridge, MA

Tarr, M. J., \& Pinker, S. (1990). When does human object recognition use a viewercentred reference frame? Psychological Science, 1, 253-256.

Tarr, M. J., Williams, P., Hayward, W. G., \& Gautier, I. (1998). Three-dimensional object recognition is viewpoint dependent. Nature Neuroscience, 1, 275-277.

Tipper, S. P., \& Behrmann, M. (1996). Object-centred not scene-based visual neglect. Journal of Experimental Psychology: Human Perception and Performance, 22, 1261-1278.

Tipper, S. P., Driver, J., \& Weaver, J. (1991). Object-centred inhibition of return in visual attention. Quarterly Journal of Experimental Psychology, 43A, 289298.

Vecera, S. P., Behrmann, M., \& Filapek, J. C. (2001). Attending to the parts of a single object: part-based selection limitations. Perception and Psychophysics, 63, 308-321.

Wetherford, M. J., \& Cohen, L. B. (1973). Developmental changes in infant visual preferences for novelty and familiarity. Child Development, 44, 416-424. 


\section{Footnotes}

1. 'Retinocentric', 'egocentric', 'allocentric' \& 'object-centred' refer to modes of spatial reference which define locations within respectively; the retina, the body, the environment, and an object. It is the independence of these reference frames from one another which makes each type of spatial reference a specific and separate encoding problem.

2. Like Shepard \& Metzler (1971), Tarr and Pinker (1990) appeal to an 'analogue mental imagery' account of object recognition. Others (e.g. Hummel, 2001; Olson \& Bialystok, 1983; Pylyshyn, 1981) have rejected this doctrine of the mental image, arguing that objects can be matched across differing orientations by the formation of spatial predicate representations of the degree of disorientation between particular common features of objects, and then comparing values of disorientations between feature pairs. This particular debate is beyond the scope of the current article. However, we can note that both strategies for recognition deal with the formation of representations of object transformation relative to the egocentric array.

3. Note that this work does not address the issue of object-centred spatial reference discussed in this manuscript, because Rochat \& Hespos (1996) do not manipulate the location of features within the rotating object.

4. Nonetheless, during the familiarisation phase, the object did undergo dynamic reorientation in full view of the infant participants. We thank an anonymous reviewer for highlighting the possibility that this dynamic presentation played a role in the infants' encoding of the spatial layout of the object, supporting their later discrimination of novel and familiar object-centred configurations. 
It would certainly be worthwhile in future research to determine whether such dynamic context is a prerequisite of the abilities demonstrated here. 


\section{Tables}

Table 1: Length of time taken for infants to accumulate the fixed looking time required in the familiarisation phase shown across three trial blocks.

Table 2: Mean percentage novelty preference as a function of age-group and dimension group.

Table 3: Mean percentage novelty preferences shown at test in Experiments 2a and $2 b$. 
Figure Captions

Figure 1: The T-shaped object

Figure 2: Spatial representations needed for making 1D and 2D discriminations of location within the T-shaped object. In the $1 \mathrm{D}$ discrimination, whichever objectcentred spatial axis is used to encode locations, the lit locations (in this example OCLs 1 and 3) hold distinctive spatial relations to other parts of the object within that axis. On axis y, OCL 3 does not have a unique value, as unlit OCL 2 shares the same value. However, OCL 1 has values on each of the axes $\mathrm{x}$ and $\mathrm{y}$ that are not shared by either of the other two locations, and so can be discriminated from OCLs 2 and 3 by reference to either axis y or $\mathrm{x}$ alone. In the $2 \mathrm{D}$ discrimination, OCLs 2 and 3 need to be encoded by reference to both axes ( $\mathrm{x}$ and $\mathrm{y}$ ). It is necessary to first note that the lit OCL is within the long bar, i.e. has a particular value on the y axis; it is then necessary to register its value on the $\mathrm{x}$ axis, defining which end of the bar it occupies.

Figure 3: The orientation series' in the familiarisation phase of Experiment 1, and the corresponding test trial arrangements. Participants presented with Series 1 during familiarisation were presented with Test Orientation 1, and participants presented with familiarisation Series 2 were given Test Orientation 2. As well as showing the orientation series used in Experiment 1 this figure also provides a schematic example of where the OCLs might have been highlighted in a stimulus presentation across a single testing session. In this case the Familiar OCL is 3, and the Novel OCL is 2 (in the $1 \mathrm{D}$ comparison) or 1 in the $2 \mathrm{D}$ comparison. Only single test trials are presented here. In all cases the novel stimulus is presented on the left. However, in an experimental session novel appeared on both the left and right on subsequent test trials. The order of left/right presentation was counterbalanced across infants. 
Figure 4: Relation between novelty preference and familiarisation duration. Dotted lines represent the $95 \%$ confidence interval.

Figure 5: The possible sequences of orientations in the familiarisation phase of Experiment 2b. As well as showing the orientation series used in Experiment $2 b$ this figure also provides a schematic example of where the OCLs might have been highlighted in a stimulus presentation across a single testing session. In this case the Familiar OCL is 3, and the Novel OCL is 1 . Only single test trials are presented here. In all cases the novel stimulus is presented on the left. However, in an experimental session novel appeared on both the left and right on subsequent test trials. The order of left/right presentation was counterbalanced across infants. 
Table 1

\begin{tabular}{|c|c|c|c|c|}
\hline \multirow[t]{2}{*}{ Age group } & \multicolumn{4}{|c|}{ Familiarisation trial block } \\
\hline & Trial block 1 & Trial block 2 & Trial block 3 & Total \\
\hline 4-month-olds $(\mathrm{N}=16)$ & $44.9(5.2)$ & $48.3(4.4)$ & $59.9(7.2)$ & $153.1(12.4)$ \\
\hline 8-month-olds $(\mathrm{N}=16)$ & $45.6(2.3)$ & $39.8(2.5)$ & $42.3(2.5)$ & $127.7(6.1)$ \\
\hline Total $(\mathrm{N}=32)$ & $45.3(2.8)$ & $44.0(2.6)$ & $51.1(4.1)$ & $140.4(7.2)$ \\
\hline
\end{tabular}

Note: Figures in brackets represent SE of mean. 
Table 2

\begin{tabular}{|c|c|c|}
\hline \multirow[t]{2}{*}{ Age group } & \multicolumn{2}{|c|}{ Dimension group } \\
\hline & 1 & 2 \\
\hline 4-month-olds & $48.7(4.0)$ & $49.3(2.9)$ \\
\hline 8-month-olds & $40.9(4.2)$ & $46.0(2.2)$ \\
\hline
\end{tabular}

Note: Figures in brackets represent SE of mean 
Table 3

\begin{tabular}{llll}
\hline Experiment & \multicolumn{1}{l}{ Test trial } & \\
& 1 & 2 & Total \\
\cline { 3 - 4 } EXP 2a (N=8) & $42.1(5.7)$ & $32.2(3.3)$ & $37.2(3.3)$ \\
EXP 2b (N=8) & $33.6(7.5)$ & $43.4(7.7)$ & $38.5(3.6)$ \\
\hline Total (N=16) & $\mathbf{3 7 . 9 ( 4 . 7 )}$ & $\mathbf{3 7 . 8 ( 4 . 6 )}$ & $\mathbf{3 7 . 8 ( 2 . 3 )}$ \\
\hline
\end{tabular}

Note: Figures in brackets represent SE of mean. 
Figure 1

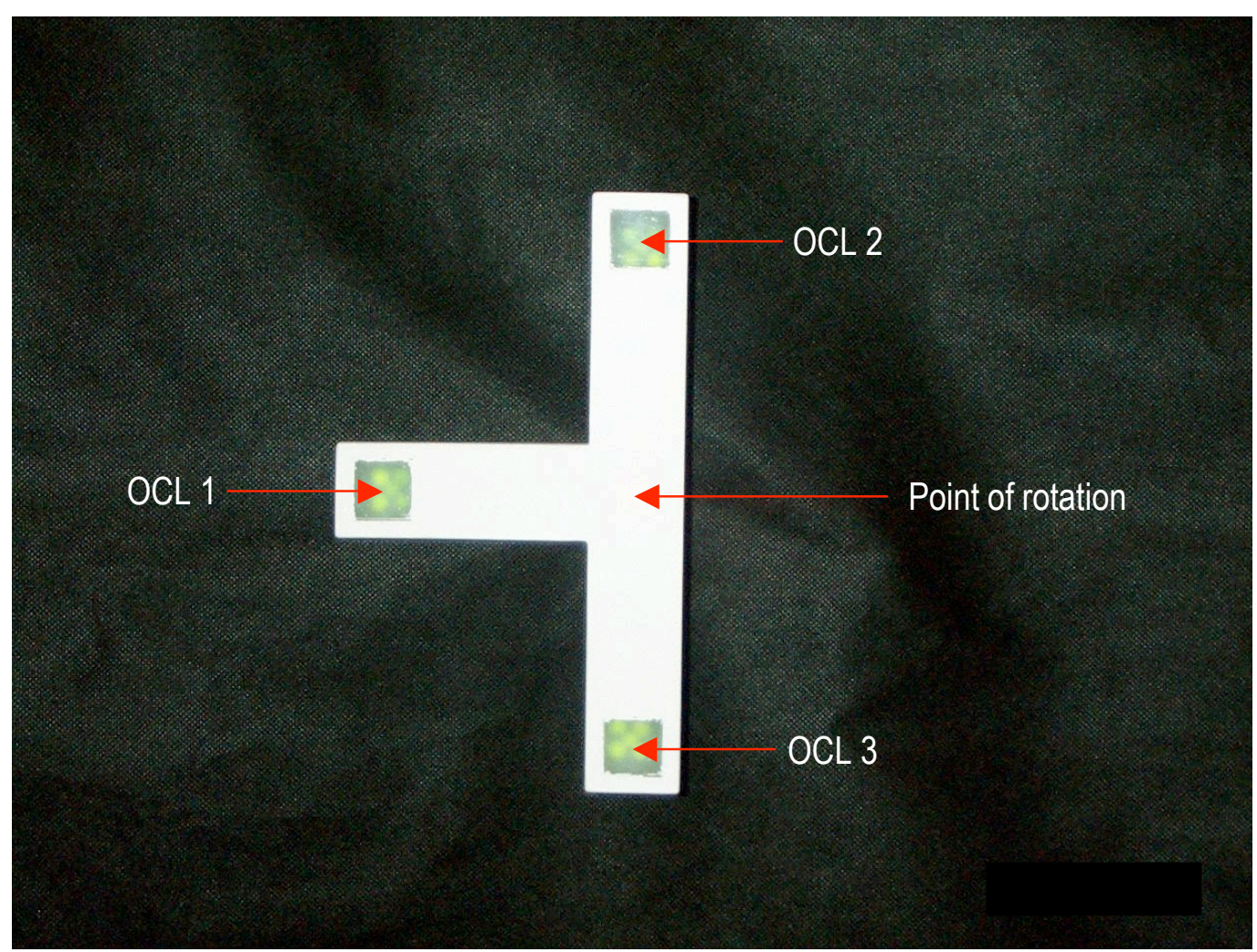


Figure 2

(A) 1D OCL discrimination
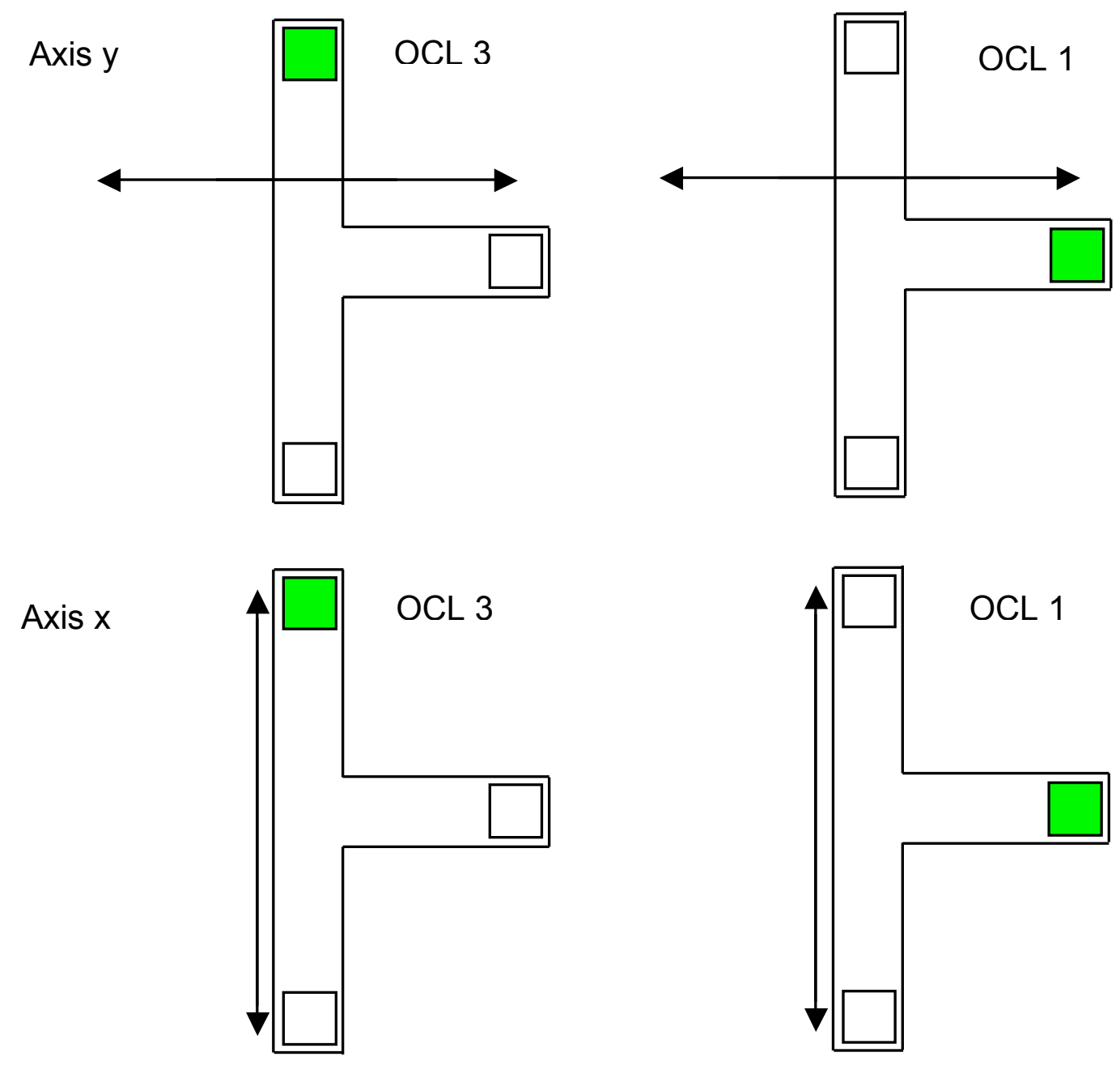

(B) 2D OCL discrimination
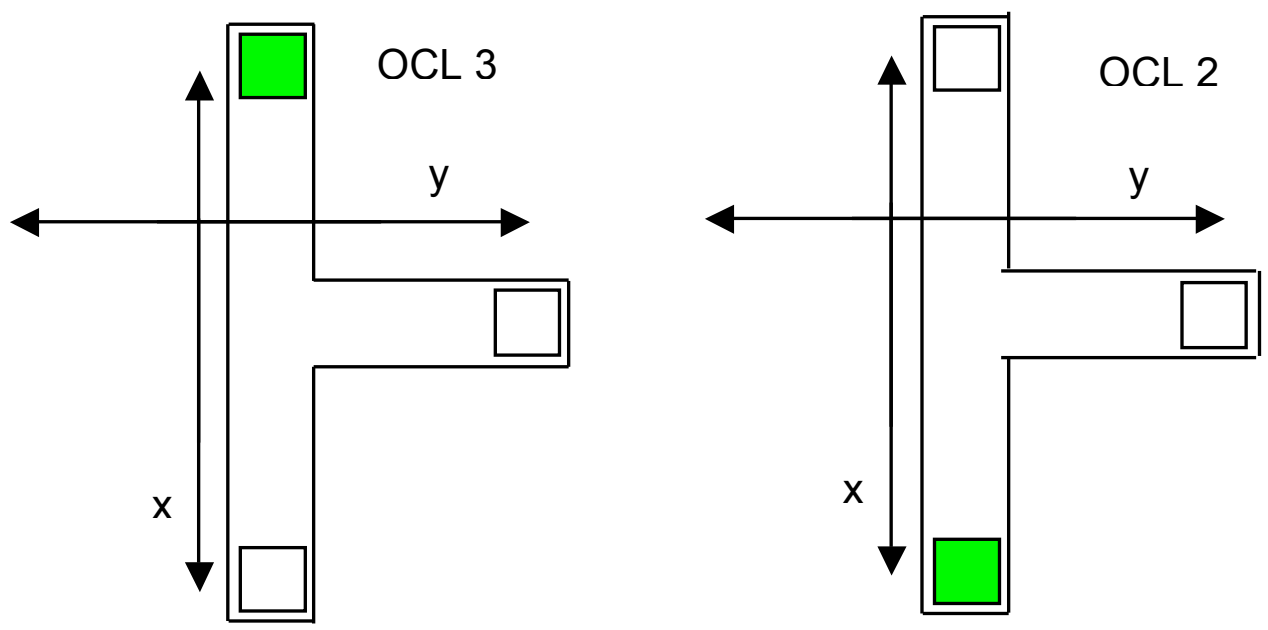
Figure 3

Familiarisation Series 1

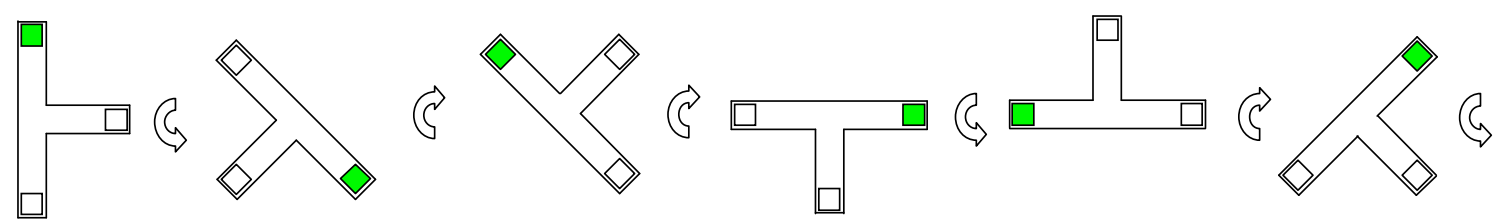

Familiarisation Series 2<smiles>O=CCCCCCCl</smiles>

Test orientations

1. (1D)

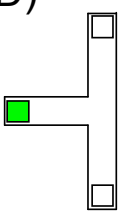

$\stackrel{\square}{\square}$

(2D)

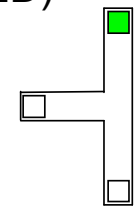<smiles>O=CC=O</smiles><smiles>CC=CCl</smiles>

$G$<smiles>ClCCCl</smiles><smiles>O=CC=CC=O</smiles>

2. (1D)
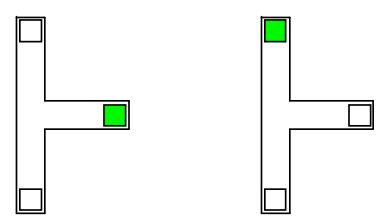

(2D)

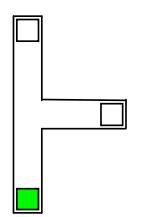<smiles>ClCCl</smiles> 
Figure 4

(A) 4-month-olds

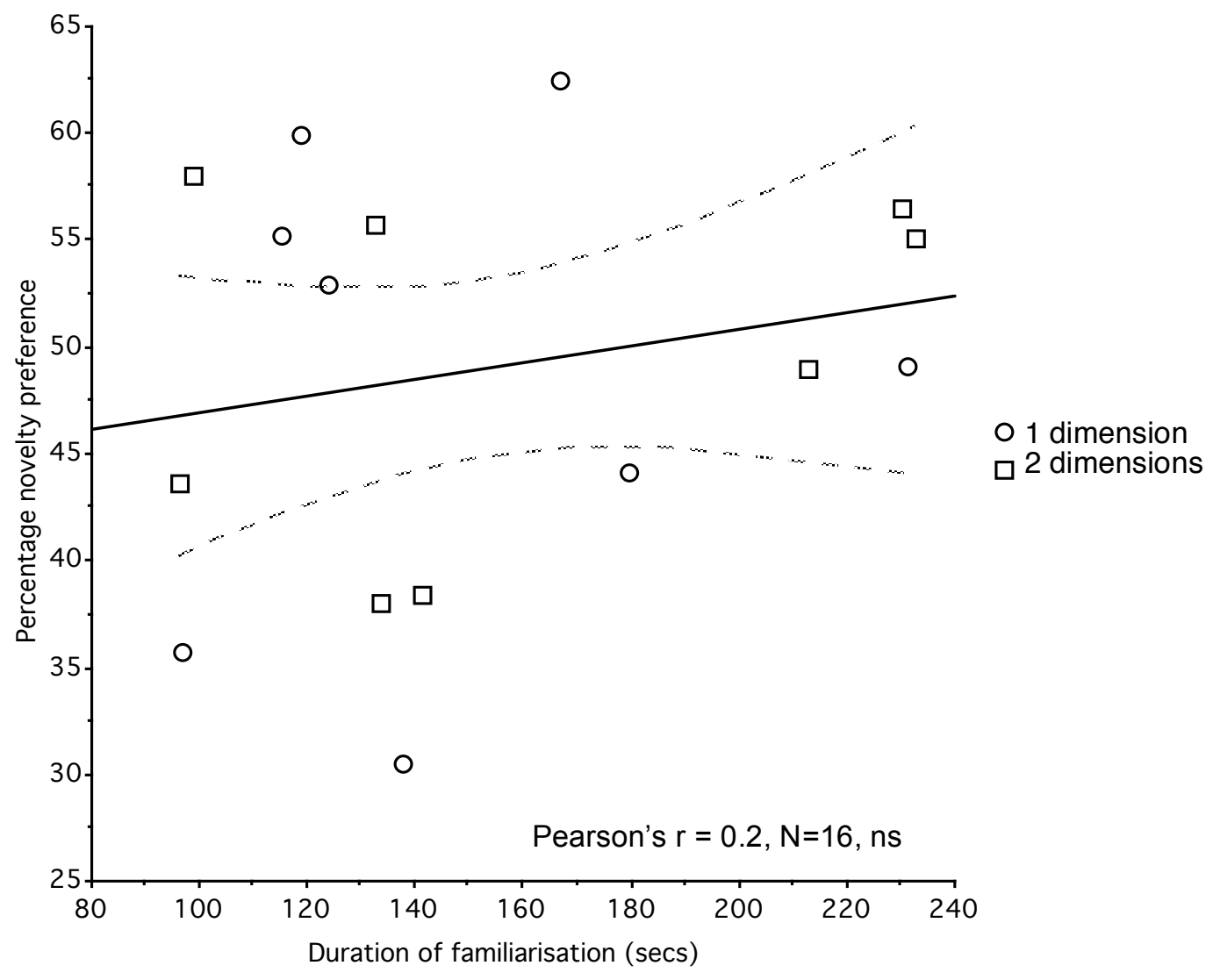

(B) 8-month-olds

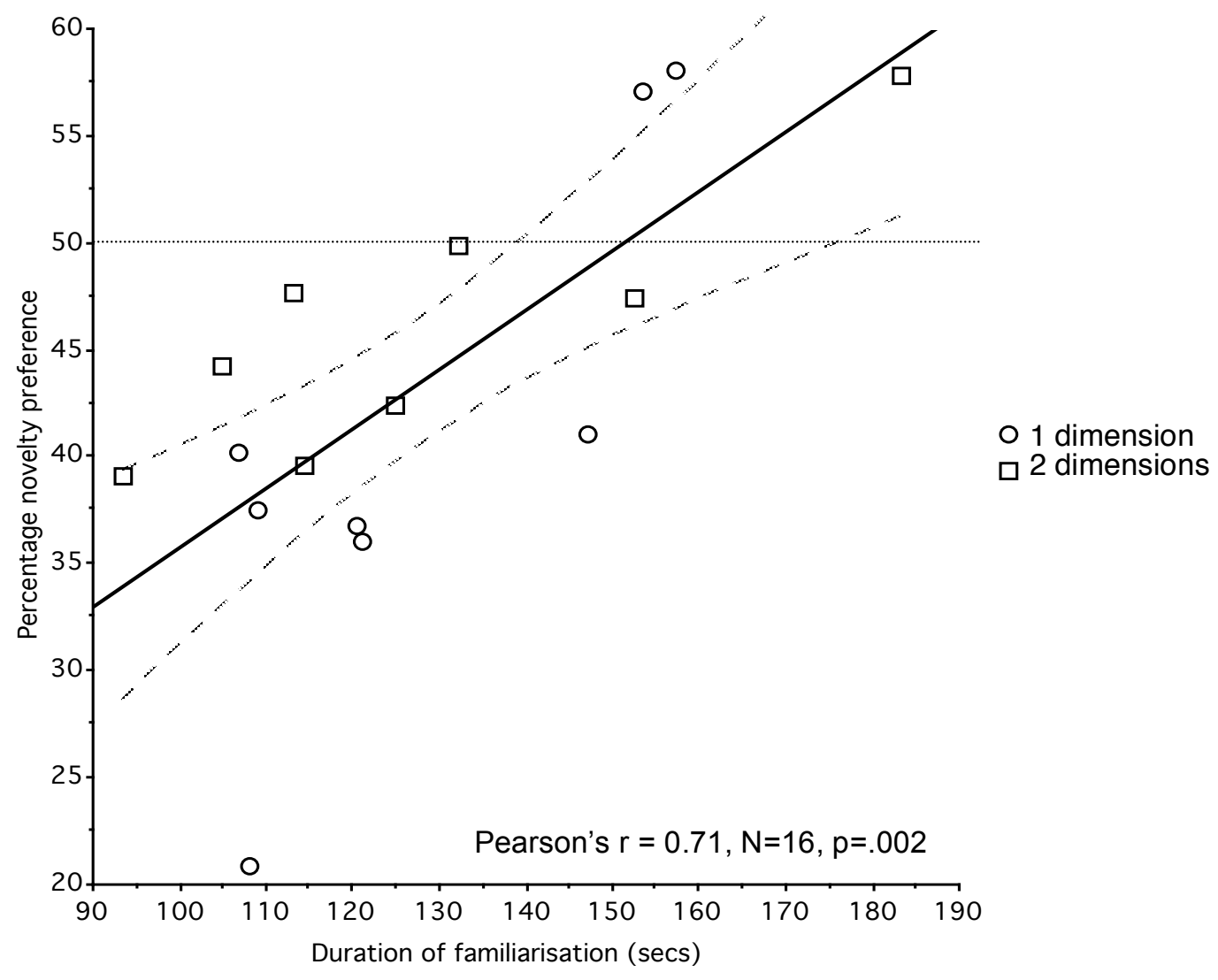


Figure 5

Starting orientation 1

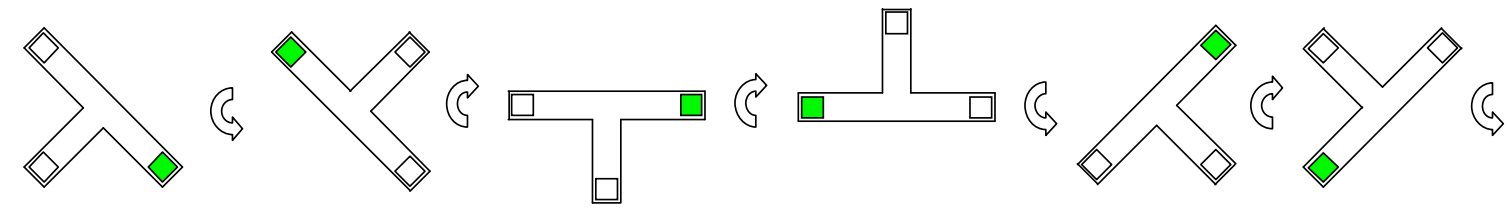

Starting orientation 2

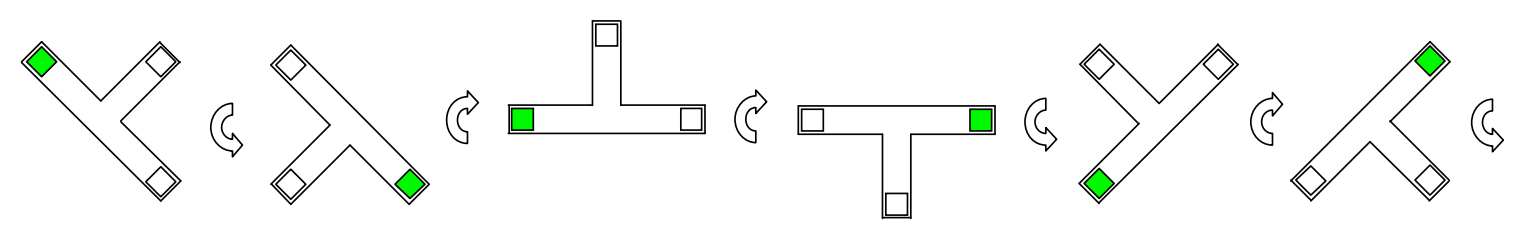

Test trial arrangements

1.

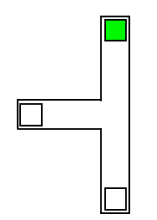

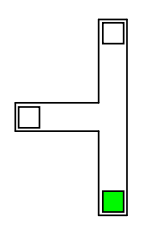

2.

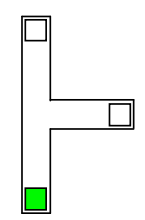

